\title{
MODELING SIMULATION AND ANALYSIS OF ROBOT GRASPING POSE FOR NOVEL OBJECTS
}

\author{
Dhanasekar.J $\mathbf{J}^{\mathbf{1}}$, Sengottuvel.P $\mathbf{P}^{\mathbf{2}}$ \\ ${ }^{1}$ Research Scholar, Department of Mechatronics, Bharath Institute of Higher Education and \\ Research, Chennai - 600 073, Tamilnadu, India \\ ${ }^{2}$ Department of Mechatronics, Bharath Institute of Higher Education and Research, Chennai - 600 \\ 073, Tamilnadu, India \\ ljdhanasekar81@gmail.com, ${ }^{2}$ dr.p.sengottuvel@gmail.com
}

\begin{abstract}
Modern day robotics aims at bringing precision and motor dexterity of humans to machines. Many scientists are working on mimicking the attributes of a human in order to grasp different object. Initially, the robots were preprogrammed to hold different object but this method is not as effective against a novel object because the image of a novel object does not match with the pre-stored program data. Hence end effector will not be able to pick up the object or it may get damaged. In this paper, a new method is discussed on the dimension and the center of mass. The robot automatically calculates the grasping point and finds the suitable pose of the gripper to grasp the desired object. This proposed work of robot simulation is achieved using MATLAB 8.2 and Creo parametric 2.0. The robot model designed using Creo is fed as input into the MATLAB to generate control signals for the robot to grasp novel objects. By this the robot will automatically grasp a new unknown object by comparing the database already created and be able to handle the object dynamically.
\end{abstract}

Keywords: D-H Transformation, Grasp points, Novel object, Robot pose, Robot simulation.

\section{Introduction}

The research of object grasping and manipulation becomes formidable challenging area [1]. The research on grasp, manipulation analysis and planning has to be applied to the real world situations. Hence increasing the efficiency of grasping and new methods is to be implemented to perform these analysis and planning tasks [2]. Grasping is one of the most fundamental problem in robotics. Robot grasping is a major problem that includes control, planning and tendency to judge the pickup points of various objects. In unstructured environments, familiar types of objects may contain a variety of shapes and sizes. Even with the existence of these variations, humans can learn how to grasp objects by few examples and generalize the learned skills to grasp novel objects [3].
However, a robot in an unstructured environment may encounter problems regarding to objects because of priori experience or knowledge. On such situations a robot requires sophisticated perception, planning and control to grasp an object [4]. Grasping planning can be classified into knowledge based, behavior based and modelbased methods [5] [6]. In this paper, we are planning to do knowledge-based grasping. Detailed knowledge is important for manipulating and grasping. The function of a robot gripper is to grasp and manipulate the object by its fingers [7]. The task of restraining objects, sometimes called fixturing and the task of manipulating objects with fingers is called dexterous manipulation [8]. From the image of an object, a location for good grasping is identified and every different 
object can have some similar subparts. [9]. A wide variety of novel object has been tested by grasping knowledge base approach and the result establishes the effectiveness of this approach so as to achieve quick and good grasps of novel objects. Grasping knowledge will enrich and serve as a best experience towards robot gripping [10], if the testing takes place with many different novel objects.

The robot with a camera will determine the center of mass and dimension of an unknown to get grasped. The object's shape and dimensions are scanned and calculated by using the camera. An algorithm is provided to predict the structure of an unknown object. A programmed robot can easily grasp object but difficult to grasp a novel object hence the friction between the gripper and the object also checked in order to avoid the damage to the objects [25-28]. In a case, the robot is performing the pick and place operation for the objects which are preprogrammed and suddenly a different object is placed, then the robot will visualize the object through camera and search for the algorithm in the database and finally executes it. If there is no data found about the object in the database, then the robot will execute the work by the combination of different objects in the database [29-34]. Here the work is carried out using a Twisting Rotation Linear and Rotation (TRLR) robot. Barrett hand is three fingered programmable grippers with ten degrees of freedom, out of which four for longer manipulation and six relatives to the object [11].

A simulator has facilities to model a complete robotic work in visualization capabilities, which is very essential when planning an actual grasping task. The real time vision system and model-based vision system are combining both to bring in a line, the pose of a simulated object with the pose of an actual object in the workspace. A simulator is used to plan, execute and monitor a complete grasping task [12]. Simulation is the imitation of operation of any machine or any system by virtual image. A robotic simulation is an imitated combination of robot motion and its operation. The simulator is software used to simulate the actual robot in a real-world environment exposed to real working conditions [35-39]. The effective design of modeling and simulation is to be tested with different robotic hand for a typical task. A Realistic simulation can be used to learn about grasping if the computer hardware model developed is better [11]. The simulation and design of the robot is carried out using MATLAB and Creo respectively. The designed model from the Creo is converted into XML file and imported into MATLAB. Then using Simulink and simmechanics, all the control algorithms for suitable grasp pose are generated for the simulation.

\section{Mechanical Design}

Barrett hand consists of disc and links as fingers. Each gripper has to be designed, fabricated for each part shape and part orientation. A robotic arm is exchanged with turret or tool changer. The tool changer took more work space and increase the production time[40-43]. The Barrett hand with complex software routine swaps the tools and parts within half a second. Figure 1 shows rotating disc of Barrett hand, Figure 2 shows Barrett finger links and Figure 3 shows the assemble view of Barrett hand.

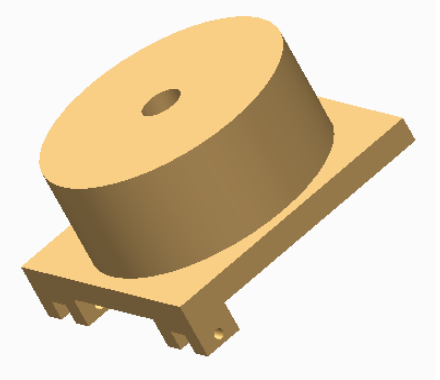

Figure 1. Rotating Disc of Barrett Hand 


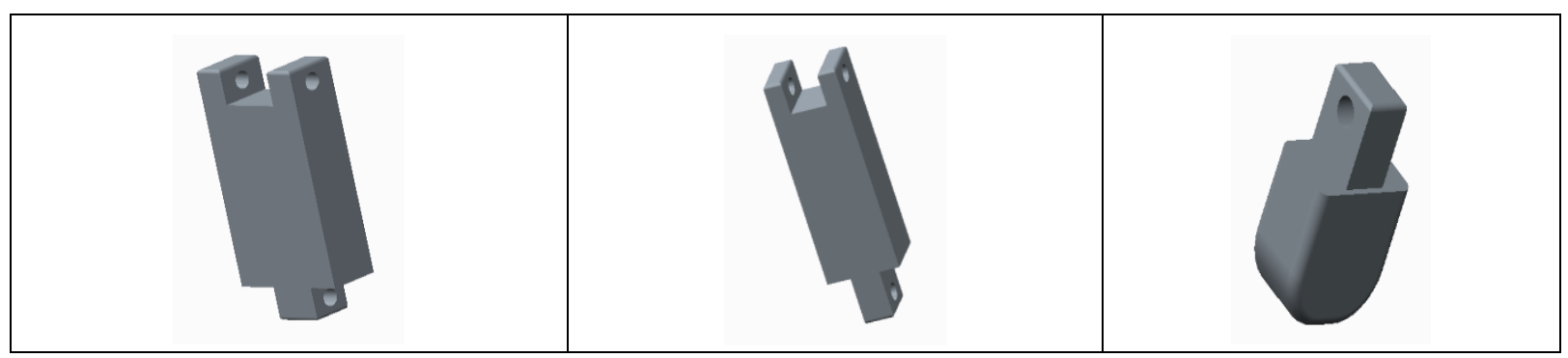

Figure 2. Barrett Finger Links

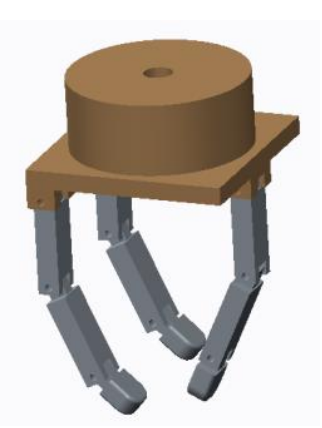

Figure 3. Assemble View of Barrett Hand

\section{Modeling Environments}

One should know the best grasping points and the angle for the object to be successfully grasped [18]. The algorithm must calculate an optimum grasping pose to feel a good grasp without collision as quick as possible [19]. Sometimes problem may occur due to miscalculation of relative position and posture between hand and the object. To overcome it, adjustment methods are proposed and simulation of the same is done to show the improvement in grasping [23].

Generally, a basic object model consists of cylinders, cones, spheres and boxes with advanced computing strategies create much number of grasp possibilities to result in high quality grasping of an object [17]. The modeling and simulation of the system highly helps in reducing time, cost and improves its efficiency of prototype. Saxena et al., (2008) proposed a learning approach which is used to predict good grasping point for a wide range of objects. In their learning approach, the 2-d location of the grasp in an object image is predicted and the projection of a good grasping point on image plane is identified. Then the images of different position of the object by different camera position are taken. After that the 3-d position of a grasping point is predicted. If both the prediction points are identified perfectly then "triangulate" these images to obtain the 3-d grasping point [9]. The camera is used to determine the pick points of an unknown object and the robot will calculate the angle, coordinate it to execute the work. However modeling is essential for us to interact with the reality which has been achieved by assumptions and testing. The scientists play an important role to design a model and to execute it accurately. The design of the robot is created using Creo software which has been further been exported to MATLAB in the form of XML file to carry the work. The top view of modeling environment is shown in Figure 4 


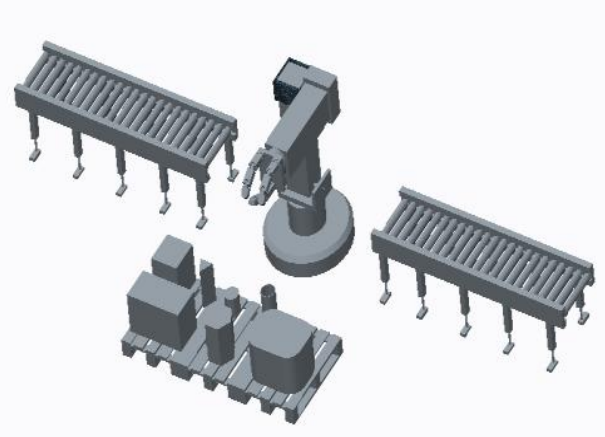

Figure 4. Top Views of Modeling Environment

Figure 5 shows the modeling of Barrett hand with objects in Creo parametric 2.0.

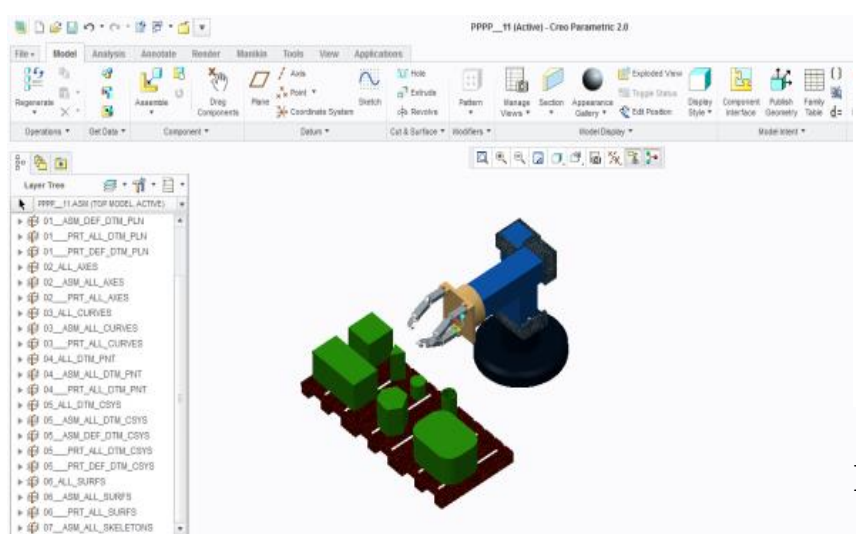

Figure 5. Modeling in Creo parametric 2.0

\subsection{Flow Chart}

A supervised training is used by robotic hand to learn good grasp. This method allows trying a large number of grasps of an object and report into training set. From the training set, generate basis functions of both, the new set of grasping parameters to be predicted and to find an optimal set of grasping parameters for an object [24]. The work flow chart is shown in Figure 6.

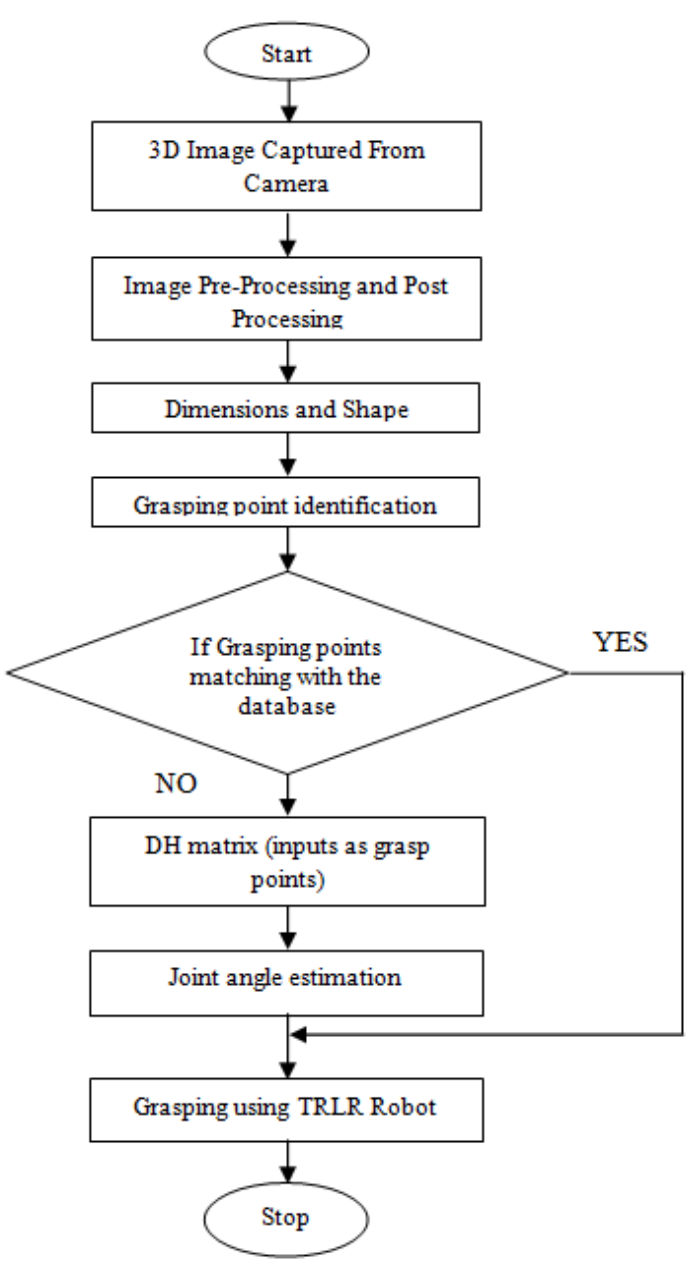

Figure 6. Work Flow Chart

The image captured by the camera will be $3 \mathrm{D}$ based. Once the image is captured, it will be preprocessed and post processed for identifying its specifications like dimensions and shape. After the identification of the shape and dimension, the grasping point will be found. The grasping point is compared with the database (already prepared one) values. If the database value matches with the grasp point, the grasp is done using TRLR robot. If it doesn't match, the D-H matrix values calculated will be considered as grasp points and the joint will be estimated. After the estimation, grasping is done by the TRLR robot.

\subsection{Grasp Point Identification}


An object detection method requires indepth offline training step for every object in order to recognize it in new images. The training process depends on every method, but getting as much information as possible is essential to create a robust object model [21]. The application of deep learning is required to detect similar problems associated with grasping and to learn the context features [15].

Three main properties of grasping were considered. First, the grasp should be able to achieve good contact with object. Second, the grasp should be stable. Third, the grasp must be able to apply forces on the object effectively [16]. Figure 7 shows the block diagram of grasp point identification. The grasp point identification is been carried out in following steps:

i. Data preprocessing: - The data preprocessing is nothing but to determine the data points of the object.

ii. Camera: - The camera is used to scan the object and it will determine the centre of gravity and centre of mass of the object

iii. Grasp point identification: - Based on the centre of gravity and centre of mass the pick points are calculated in grasp point identification.

iv. Processing: - By calculating this pick points the work can be executed.

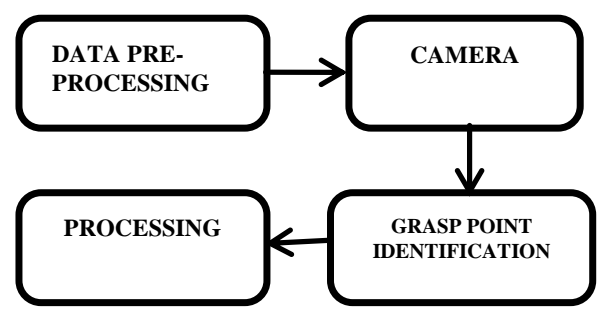

Figure 7. Block diagram of grasp point identification
Generally, the robot grasping varies with respect to the perception of human and the robot. Without training the grasping may be inaccurate with the robot. To overcome these grasping problems the software and camera arrangement were introduced. The grippers are well designed such that it should not damage the object. To have a stable grasp, the algorithm should be accurate to determine the pick points of the novel objects. And the camera should have the capability to judge the shape and dimensions of the object to execute the operation accurately. By executing all these things, it can perform the work perfectly without any errors or collision in the system.

The grasp point detection finds the surface of all models with a definite threshold and generates 2D information then gets the rim points and edges of the object from the details. Then calculate the centre of mass for every object [20]. To generate observation space for grasping the object, probabilistic models can be used. A probabilistic model helps to learn about the task constraints in robot grasping [13]. The segmentation of an unknown object will be achieved with a 3-d mesh generation. The robotic grasping depends upon the shape and dimension of the object. And the grasp pose for the TRLR robot will be determined using the inverse kinematics. This helps the robot to detect the angle position to grasp the object. As per the conventional stability, to get a stable grasp the pick points should be near to the centre of mass of the object. We intersect the point with horizontal planes through the centre of object. With these $n$ cutting plane points $p i$, we calculate the convex hull V. The expressions are shown in Equation (1). The distance between two hull points to the centre of the object is c and we calculate the altitude $\mathrm{d}$. where $\mathrm{v}$ is the direction vector to the two neighboring hull point and $\mathrm{w}$ is the direction vector to c. Finally, the algorithm finds the shortest normal distance "d" of the convex hull line. 
(1)

$$
d=\frac{\|\vec{v} * \vec{w}\|}{\|\vec{v}\|}
$$

The second grasping point is been determined by the reflection of the first grasping point using the centre of the object. We can check the grasp of the object on the detected grasp points with a 3D model. Figure 8 shows the grasp points of various objects.

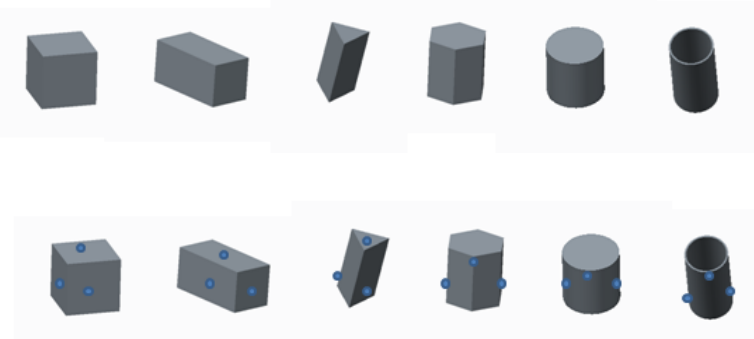

Figure 8. Grasp Points

\subsection{Grasp Pose}

RajeshkannaAla et al., (2015) proposed an algorithm providing a way to grasp any object using boundary, envelope, and functional grasps. This algorithm is based on identifying graspable segments, analyzing geometrically and incorporating the memory of grasping experience [14]. A grasping algorithm also decides at which angle it has to grasp the object. The robot works in the inverse kinematics method. After detecting the object, the robot will look for the pick points through which the coordinates are determined. With the help of these coordinates the robot will decide in which angle the object is to be picked and placed. The tabulations for D-H parameters are shown in Table 1 and Figure 9 shows grasp pose view.

Table 1. D-H Representation

\begin{tabular}{|l|l|l|l|l|l|}
\hline $\mathrm{A}$ & $\#$ & $\theta$ & $\mathrm{d}$ & $\mathrm{a}$ & $\alpha$ \\
\hline $\mathrm{A}_{1}$ & 1 & $\theta_{1}$ & 0 & 0 & 90 \\
\hline $\mathrm{A}_{2}$ & 2 & $\theta_{2}$ & 0 & $\mathrm{a}_{2}$ & 0 \\
\hline $\mathrm{A}_{3}$ & 3 & $\theta_{3}$ & 0 & $\mathrm{a}_{3}$ & 0 \\
\hline $\mathrm{A}_{4}$ & 4 & $\theta_{4}$ & 0 & $\mathrm{a}_{4}$ & -90 \\
\hline
\end{tabular}

\begin{tabular}{|l|l|l|l|l|l|}
\hline $\mathrm{A}_{5}$ & 5 & $\theta_{5}$ & 0 & 0 & 90 \\
\hline $\mathrm{A}_{6}$ & 6 & $\theta_{6}$ & 0 & 0 & 0 \\
\hline
\end{tabular}

The final transformation matrix is given by Eq. (2)

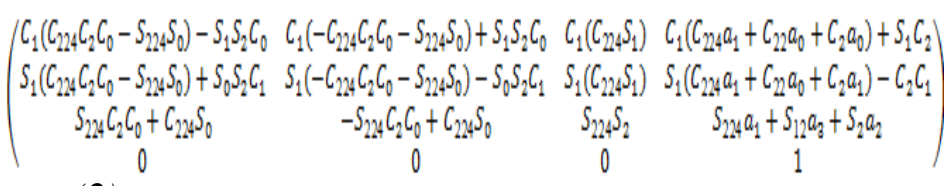

(2)

Alternatively, Equation (2) expressed with trigonometric function Equation (3) and Equation (4)

$$
\mathrm{S} \theta_{1} \mathrm{C}_{2}+\mathrm{C} \theta_{1} \mathrm{~S} \theta_{2}=\mathrm{S}\left(\theta_{1}+\theta_{2}\right)=\mathrm{S}_{12}
$$

$$
\mathrm{C} \theta_{1} \mathrm{C} \theta_{2}-\mathrm{S} \theta_{1} \mathrm{~S} \theta_{2}=\mathrm{C}\left(\theta_{1}+\theta_{2}\right)=\mathrm{C}_{12}
$$

(4)

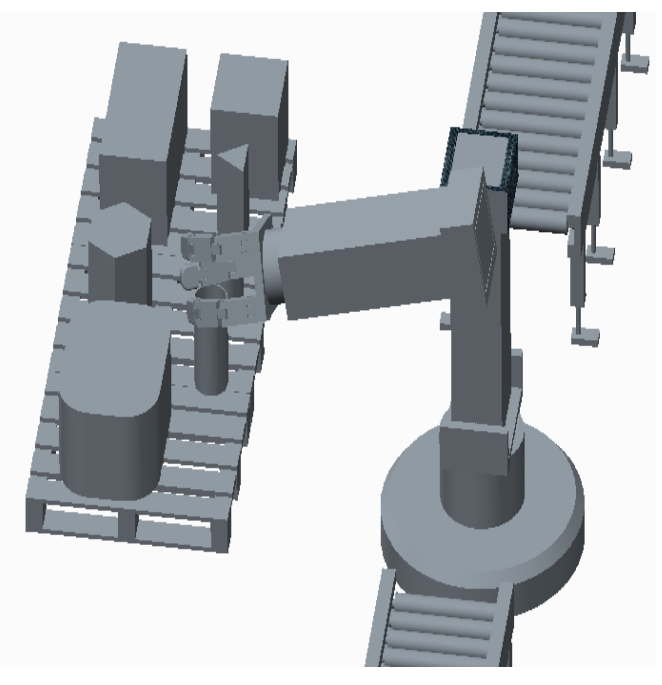

Figure 9. Grasp Pose View

\subsection{Experimental Setup for Grasping of Novel Object}

When modeling an object as a set of shape primitives, such as cones, spheres, cylinders and boxes, we can utilize a set of rules to generate a set of grasps starting positions and pre-grasp shapes that can be tested on the object model [22]. Figure 10 shows the grasping setup of known object. This setup consists of base, frame, pillar, 
gripper and two cameras. The snap of the inline objects has taken using the cameras and then compared with the database which consists of grasping algorithm for standard models like cylinder, cone, sphere and box. If the particular shape matches with the database then it commands the TRLR robot to do the operation.

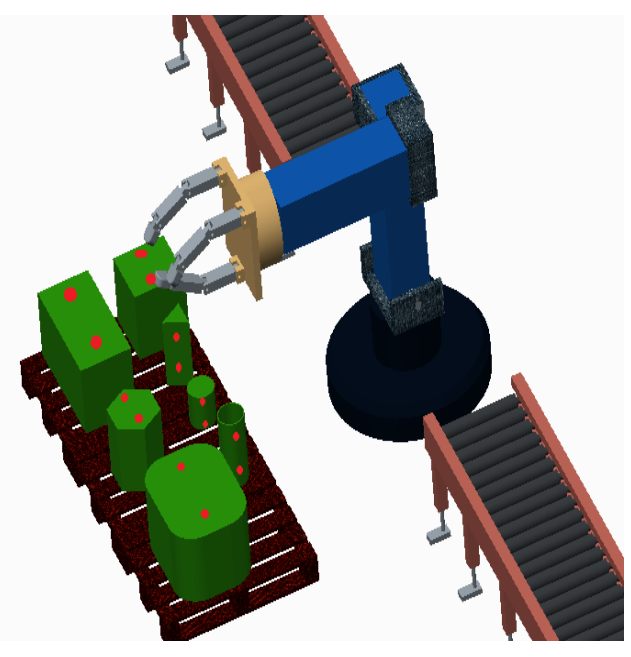

Figure 10. Grasping setup of known object
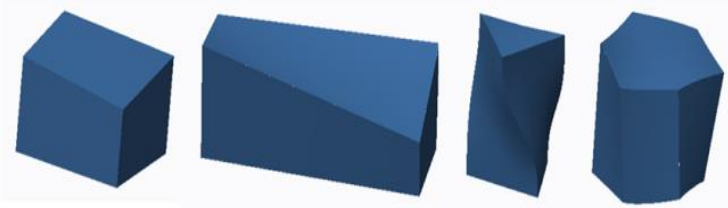

\section{Figure 11. Novel objects}

Figure 11 shows some novel objects to be grasped. If the unknown or novel object presented does not match with the database, then with the MATLAB tool box, the robot finds out the bounding box and centre of mass value for the novel object. The bounding box value of the novel object close to the standard model value helps to choose an algorithm for that particular shape. Then the D-H matrix of that shape is found to form the kinematic equation of the manipulator. From the equation the joint angle is then estimated and the TRLR robot is given command to pick the novel object.

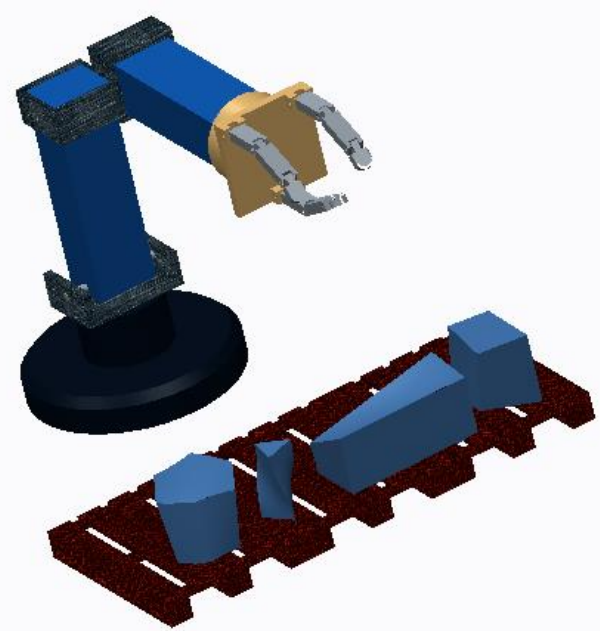

Figure 12. Setup of robot with novel objects

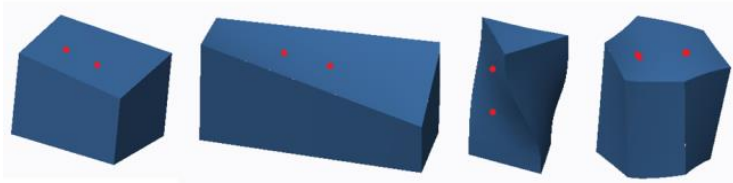

Figure 13. Grasping point of novel objects

In Figure 12 shows the setup of robot with novel objects. Grasping points for the novel objects is shown in Figure 13. Figure 14 predicts the grasping setup of novel objects with grasping points.

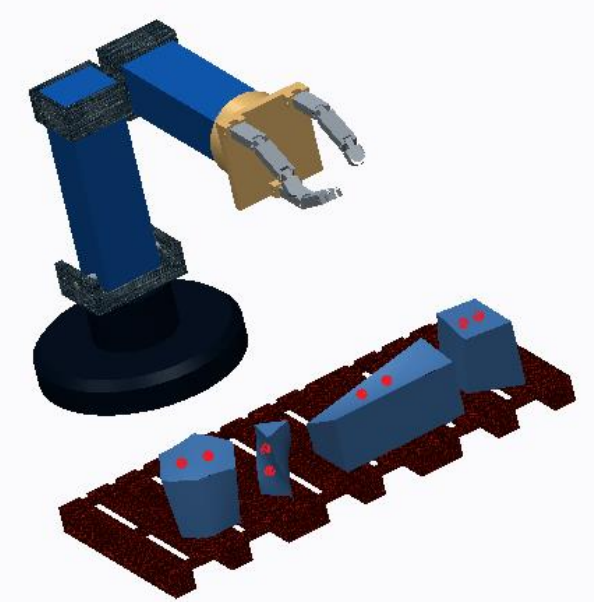

Figure 14. Grasping setup of novel objects with grasping point 
The grafpraspsetup of novel $\phi$ bjects with

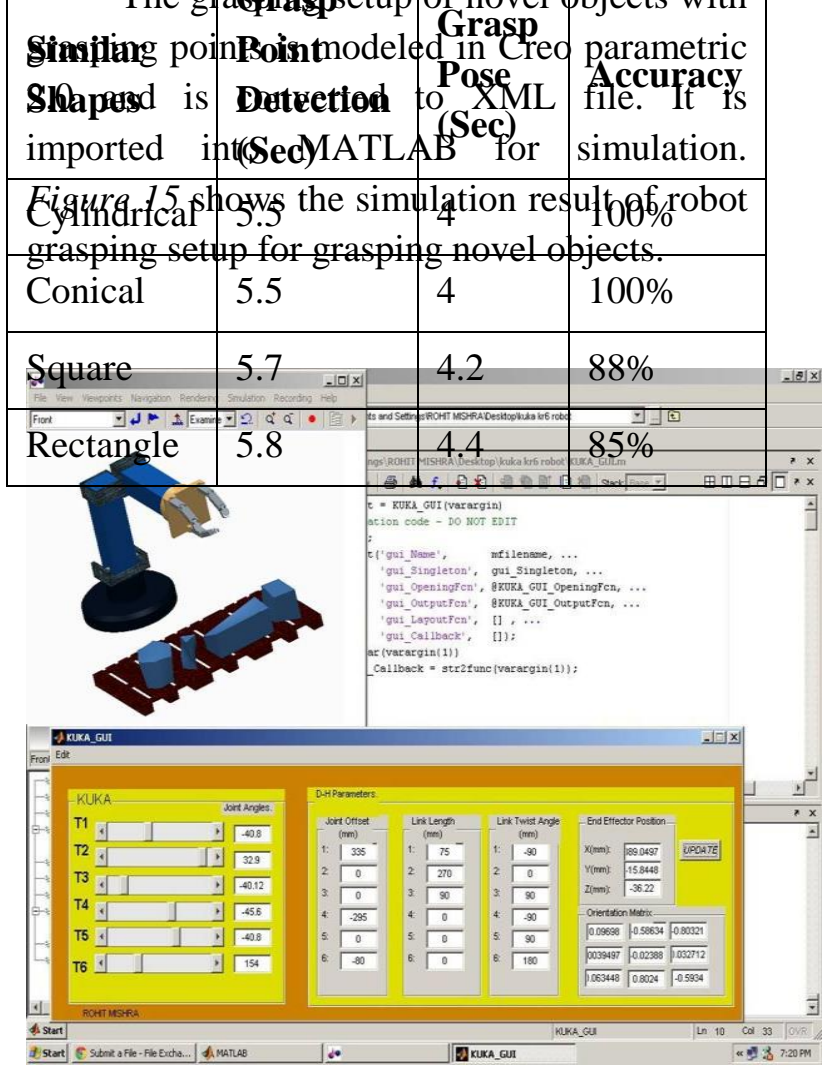

Figure 15. Simulation of robot grasping in MATLAB

\section{Result and Discussion}

The simulation experiment has been carried out for similar geometric shapes like conical, cylindrical, square and rectangle. The average time taken for grasp point detection is about $5.5 \mathrm{sec}$ and for grasp pose is $4 \mathrm{sec}$. The success rate of grasping similar cylindrical and conical shape is $100 \%$. For square and rectangular shape is $88 \%$ and $85 \%$ respectively. The tabulations for grasp point detection and accuracy are shown in Table 2.

\section{Table 2. Grasp Point Detection and} Accuracy

The algorithm requires less simulation time for preprogrammed similar geometrical shapes. In case of irregular shape, grasping accuracy is very less and simulation time is more.

\section{Conclusion}

Based on the camera image and the image processing techniques the dimensions and shape of the object is calculated. From the dimensions and shape results, suitable grasp points are generated successfully. Now the grasp points (assumed as coordinates in 3D space) are given as inputs to the TRLR robot. Based on the D-H matrix results for inverse kinematics are calculated which gives the joint angles for the TRLR robot. All these practices are performed using MATLAB simulation. A simple neural based learning algorithm has been implemented and tested to grasp novel objects, which fails in several practical applications. But this system is suitable only for rigid bodies. This algorithm/method does not fit for deformable objects i.e. objects which change its shape, dimensions and mass with respect to time. Another important drawback is that mass is considered as a constant in this system.

\section{References}

1. D. Song, K. Huebner, V. Kyrki, and D. Kragic, "Learning Task Constraints for Robot Grasping using Graphical Models," in IROS, 2010, pp. 15791585 .

2. C. Borst, M. Fischer, and G. Hirzinger, "Grasp Planning: How to Choose a Suitable Task Wrench Space," in Proceedings of the IEEE Int. Conf. on Robotics and Automation, 2004, pp. 319-325.

3. A Boularias et al., Learning Robot Grasping from 3-D Images with Markov Random Fields, 2011 IEEE/RSJ International Conference on Intelligent Robots and Systems. 
4. Pei-Chi Huang et al., Grasping Novel Objects with a Dexterous Robotic hand through Neuroevolution, IEEECICA, doi: 10.1109/CICA.2014.7013242, 2014.

5. B. Wang, L. Jiang, J. LI, H. Cai, Grasping unknown objects based on 3D model reconstruction, Advanced Intelligent Mechatronics. Proceedings, 2005 IEEE/ASME International Conference on (2005) pp. $461-466$.

6. B Wang et al., Grasping Unknown Objects Based on 3D Model Reconstruction, Proceedings of the 2005 IEEE/ASME, International Conference on Advanced Intelligence Mechatronics, 2005, pp. 461466.

7. A Sahbani, et al., An overview of 3D object grasp synthesis algorithms, Robotics and Autonomous Systems (2011), doi: 10.1016/j.robot.2011.07.016.

8. Bicchi and V. Kumar, Robotic grasping and contact: a review, in: IEEE International Conference on Robotics and Automation, vol. 1, 2000, pp.348-353.

9. A Saxena et al., Robotic Grasping of Novel Objects using Vision, The International Journal of Robotics Research, February 2008, Vol.27 no.2157-173, doi: $10.1177 / 0278364907087172$.

10. N Curtis at al., Efficient and Effective Grasping of Novel Objects through Learning and Adapting a Knowledge Base, 2008 IEEE/RSJ International Conference on Intelligent Robot and
Systems , Nice, France, September, 22-26, 2008.

11. Miller et al., From robotic hands to human hands: a visualization and simulation engine For grasping research, Industrial Robot: An International Journal 32/1 (2005) 5563, Emerald Group Publishing Limited [ISSN 0143-991X][DOI 10.1108/01439910510573309].

12. Andrew T. Miller, GraspIt!: A Versatile Simulator for Robotic Grasping, IEEE Robotics \& Automation Magazine, Volume 11, Issue 4, December 2004, doi: 10.1109/MRA.2004.1371616, Print ISSN: 1070-9932, pp.110-122.

13. H. Ek, D. Song, K. Huebner, and D. Kragic.Exploring affordances in robot grasping through latent structure representation. In Vision for Cognitive Tasks, ECCV, September 2010.

14. RajeshkannaAla et al., A 3D-Grasp synthesis algorithm to grasp unknown objects based on graspable boundary and convex segments, Elsevier, Science Direct, Information Sciences 295(2015) 91-106, doi:10.1016/j.ins.2014.09.062.

15. Lenz, H. Lee, and A. Saxena, "Deep learning for detecting robotic grasps," in RSS, 2013.

16. L. Wong, Learning to select robotic grasps using vision on the stanford artificial intelligence robot, Stanford Undergraduate Res. J. 7 (2008) 59-64.

17. A T Miller et al., Automatic Grasp Planning Using Shape Primitives, 2003 IEEE International Conference on Robotics and Automation, DOI: 
10.1109/ROBOT.2003.1241860, Print ISSN: 1050-4729.

18. Richtsfeld M, Vincze M (2011) Robotic grasping of unknown objects. In: Goto S (ed) Robot arms. InTech, Rijeka, Croatia, pp. 123-136.

19. Mario Richtsfeld y Markus Vincze.Robotic Grasping of Unknown Objects. INTECH Open Access Publisher, 2009, pp. 19-34.

20. Mario Richtsfeld, Markus Vincze. Grasping of Unknown Objects from a Table Top, Workshop on Vision in Action: Efficient strategies for cognitive agents in complex environments, October 2008, Marseille, France. 2008. <inria00325794>

21. F. R. Aparici, A. R. Ayats, G. A. Ribas, X. T. Genis, "Object detection methods for robot grasping: Experimental assessment and tuning," in Proc. 15th Catalan Conference on Artificial Intelligence (CCIA), 2012, pp. 123-132.

22. Miller, A. T., Knoop, S., Christensen, H. I and Allen, P. K. (2003). Automatic Grasp Planning using Shape Primitives, IEEE International Conference on Robotics and Automation, pp. 1824-1829.

23. Y Hasegawa et al., Flexible control of a grasping object with posture error, International Conference on FUZZIEEE'02, vol. 2, 2002, pp. 1550-1555, DOI: 10.1109/FUZZ.2002.1006737.
24. R. Pelossof, A. Miller, P. Allen, and T. Jebara. An SVM learning approach to robotic grasping, In International Conference on Robotics and Automation (ICRA), 2004.

25. Ganesh Babu Loganathan, Praveen M., Jamuna Rani D., "Intelligent classification technique for breast cancer classification using digital image processing approach" IEEE Xplore Digital Library 2019, Pp.1-6.

26. Dr.Idris Hadi Salih, Ganesh Babu Loganathan, "Induction motor fault monitoring and fault classification using deep learning probablistic neural network" Solid State Technology(2020), Volume 63, Issue 6, PP No. 2196-2213.

27. Ganesh Babu Loganathan, "Design and analysis of high gain Re BoostLuo converter for high power DC application", Materials Today: Proceedings(2020), Volume 33, Part 1, PP 13-22.

28. M. Viswanathan, Ganesh Babu Loganathan, and S. Srinivasan, "IKP based biometric authentication using artificial neural network", AIP Conference Proceedings (2020), Volume 2271, Issue 1, pp 030030.

29. Mohammed Abdulghani Taha and Ganesh Babu Loganathan, "Hybrid algorithms for spectral noise removal in hyper spectral images" AIP Conference Proceedings (2020), Volume 2271, Issue 1, pp 030013.

30. Ganesh Babu Loganathan, "Vanet Based Secured Accident Prevention System", International Journal of Mechanical Engineering and Technology (IJMET)(2019), Vol.10 Issue No.06, P.No. 285-291. 
31. Ganesh Babu Loganathan, "Can Based Automated Vehicle Security System", International Journal of Mechanical Engineering and Technology (IJMET)(2019), Vol.10 Issue No.07, P.No. 46-51.

32. G Sharma, A Rajesh, L Ganesh Babu, E Mohan, "Three-Dimensional Localization in Anisotropic Wireless Sensor Networks Using Fuzzy Logic System", Adhoc \& Sensor Wireless Networks, (2019) Vol.45 Issue No.1, P.No. 29-57.

33. Ganesh Babu Loganathan, Dr.E.Mohan, R.Siva Kumar, " Iot Based Water And Soil Quality Monitoring System", International Journal of Mechanical Engineering and Technology (IJMET)(2019), Vol.10 Issue No.2, P.No. 537-541.

34. Suganthi K, Idris Hadi Salih, Ganesh Babu Loganathan, and Sundararaman K, "A Single Switch Bipolar Triple Output Converter with Fuzzy Control", International Journal of Advanced Science and Technology, (2020), Vol. 29, No. 5, (2020), P.No.. $2386-2400$.

35. B.K. Patle, Ganesh Babu L, Anish Pandey, D.R.K. Parhi, A. Jagadeesh,A review: On path planning strategies for navigation of mobile robot, Defence Technology, Volume 15, Issue 4, August 2019, Pages 582-606.

36. Dr.A.Senthil Kumar, Dr.Venmathi A R ,L.Ganesh Babu, Dr.G. Suresh, "Smart Agriculture Robo With Leaf Diseases Detection Using IOT", European Journal of Molecular \& Clinical Medicine, Volume 07, Issue 09, PP 2462-2469.
37. Ganesh Babu L 2019 Influence of benzoyl chloride treatment on the tribological characteristics of Cyperus pangorei fibers based nonasbestos brake friction composites Mater. Res. Express 7015303.

38. Manoharan S, Sai Krishnan G, Babu L G, Vijay R and Singaravelu D L 2019 Synergistic effect of red mud-iron sulfide particles on faderecovery characteristics of non-asbestos organic brake friction composites Mater. Res. Express 6105311.

39. Manoharan S, Shihab A I, Alemdar A S A, Ganesh Babu L, Vijay R and Lenin Singaravelu D 2019 Influence of recycled basalt-aramid fibres integration on the mechanical and thermal properties of brake friction composites Material Research Express 6115310 .

40. G Sai Krishnan, L Ganesh Babu, P Kumaran , G Yoganjaneyulu and Jeganmohan Sudhan Raj, "Investigation of Caryota urens fibers on physical, chemical, mechanical and tribological properties for brake pad applications", Material Research Express 7015310

41. A.Devaraju, P.Sivasamy, Ganesh BabuLoganathan, "Mechanical properties of polymer composites with ZnO nano-particle", Materials Today:

Proceedings(2020), Volume 22, Part 3, Pages 531-534.

42. Mr.Manikandan Ganesan, Mrs.Ishwarya K. R, Mr. Demoz Lisanework, Mr.Ayenachew Hailu Mengiste, "Investigation On Autonomous Pesticide Spraying 
Robotic Vehicle In Agriculture Field", International Journal of Modern Agriculture, Volume 10, No.1, 2021 pp 382-386.

43. Ellappan Mohan, Arunachalam Rajesh, Gurram Sunitha, Reddy Madhavi Konduru, Janagaraj
Avanija, Loganathan Ganesh Babu, "A deep neural network learning-based speckle noise removal technique for enhancing the quality of synthetic-aperture radar images", Concurrency And ComputationPractice \& Experience, https://doi.org/10.1002/cpe.6239 